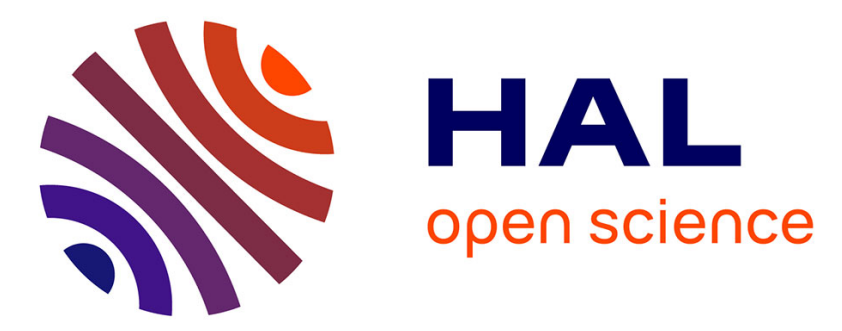

\title{
L'hérédité de la résistance au Sclerotinia sclerotiorum (Lib.) de Bary chez le tournesol. I. Etude des réactions à deux tests mycéliens
}

Nathalie Robert, Felicity Vear, Denis D. Tourvieille de Labrouhe

\section{- To cite this version:}

Nathalie Robert, Felicity Vear, Denis D. Tourvieille de Labrouhe. L'hérédité de la résistance au Sclerotinia sclerotiorum (Lib.) de Bary chez le tournesol. I. Etude des réactions à deux tests mycéliens. Agronomie, 1987, 7 (6), pp.423-429. hal-00885010

\section{HAL Id: hal-00885010 https://hal.science/hal-00885010}

Submitted on 1 Jan 1987

HAL is a multi-disciplinary open access archive for the deposit and dissemination of scientific research documents, whether they are published or not. The documents may come from teaching and research institutions in France or abroad, or from public or private research centers.
L'archive ouverte pluridisciplinaire HAL, est destinée au dépôt et à la diffusion de documents scientifiques de niveau recherche, publiés ou non, émanant des établissements d'enseignement et de recherche français ou étrangers, des laboratoires publics ou privés. 


\title{
L'hérédité de la résistance au Sclerotinia sclero- tiorum (Lib.) de Bary chez le tournesol. I. Etude des réactions à deux tests mycéliens
}

\author{
Nathalie ROBERT, Felicity VEAR $\left({ }^{*}\right)$ \& Denis TOURVIEILLE DE LABROUHE $\left({ }^{* *}\right)$ \\ I.N.R.A. Station d'Amélioration des Plantes, BV 1540, F 21034 Dijon Cedex \\ (*) I.N.R.A., Station d'Amélioration des Plantes \\ (**) Station de Pathologie végétale, Centre de Recherches de Clermont-Ferrand-Theix, Domaine de Crouëlle, \\ F 63039 Clermont-Ferrand
}

\begin{abstract}
Deux tests de sélection ont été employés sur tiges et capitules de tournesol pour estimer la résistance des tissus à l'extension du mycélium de Sclerotinia sclerotiorum. Ces tests ont été comparés à des observations réalisées lors d'infections naturelles sur cotylédons, bourgeons et capitules. L'étude a porté sur les lignées parentales et les hybrides F1 d'un plan factoriel de croisement de 6 mâles-stériles et 6 restaureurs.

La résistance est polygénique et fait intervenir des gènes différents pour chaque organe. Le test sur capitule présente le meilleur niveau d'héritabilité. L'additivité apparaît comme plus importante que les interactions de dominance. La valeur propre des lignées est un bon prédicteur de la valeur en combinaison. L'utilisation des 2 types de tests en sélection est discutée.
\end{abstract}

Mots clés additionnels : Helianthus annuus, pourriture blanche, sélection, héritabilité, additivité, dominance.

\begin{abstract}
Inheritance of resistance to Sclerotinia sclerotiorum in sunflower. I. Results of two tests with mycelial inoculum.

Two breeding tests were tried out on stems and capitula of sunflowers to estimate tissue resistance to spread of Sclerotinia sclerotiorum mycelium. These tests were compared with observations of natural attacks on cotyledons, terminal buds and capitula. The study was made on the parental inbred lines and $F 1$ hybrids of a factorial cross of 6 male steriles and 6 restorers. Resistance to $S$. sclerotiorum is polygenic and for each organ, different genes are involved. The capitulum test showed the best level of heritability. Additivity appeared more important than dominance interactions. Measurements of resistance on inbred lines provided good estimates of resistance levels in hybrids. Use of the two types of tests in breeding programmes is discussed.
\end{abstract}

Additional key words : Helianthus annuus, white rot, breeding, heritability, additivity, dominance.

\section{INTRODUCTION}

Sclerotinia sclerotiorum (Lib.) de Bary, Ascomycète présent sur tous les continents, s'attaque à la plupart des Dicotylédones et n'épargne ni les plantes maraîchères et ornementales ni les plantes de grande culture (PuRdy, 1979).

Sur tournesol, plusieurs formes de la maladie existent (TOURVIEILLE \& VEAR, 1984a) :

- attaques sur racines et collet, provoquées par le mycélium issu de la germination végétative des sclérotes ;

- attaques sur organes aériens (bourgeon apical, feuilles, tige et capitule), résultant de l'infection par les ascospores produites par les apothécies, organes de la reproduction sexuée.

L'importance relative de ces types d'attaques varie selon la zone de culture. En France, l'infection du capitule est la forme prépondérante et la plus dommageable (VEAR \& TOURVIEILLE, 1984a).

Du fait de la longue conservation de l'inoculum dans le sol, du caractère polyphage du champignon et de l'inefficacité des traitements chimiques (LAMARQUE et al., 1985), le seul moyen de lutter actuellement contre la pourriture blanche du tournesol est la création de variétés résistantes. La résistance est partielle et polygénique. L'utilisation, sur 9 hybrides, de plu- 
sieurs tests de sélection appréciant la résistance aux différentes phases de l'infection (pénétration et extension dans les tissus) a montré que les génotypes diffèrent par leur comportement à chacune de ces phases et qu'il y a indépendance entre les réactions aux différentes attaques, pour un même hybride (TOURVIEILlE \& VEAR, 1984a).

Les observations d'attaques naturelles sous irrigation et l'application de différents tests sont possibles à tous les stades de sélection. Cependant, si les notations d'attaques naturelles ne posent pas de problème sur des hybrides, elles sont difficiles sur les lignées à cause du manque de vigueur, du dessèchement rapide et de la présence de ramifications (VEAR \& TOURVIEILLE, résultats non publiés). Par contre, les tests de résistance sont assez lourds et le nombre de génotypes qui peut être étudié chaque année est relativement limité : on ne peut pas envisager de conduire ces tests sur un grand nombre d'hybrides expérimentaux. C'est au niveau de la sélection pour la résistance au $S$. sclerotiorum parmi des populations ou des descendances en ségrégation ou pour l'étude de lignées fixées que ces tests paraissent intéressants.

La mise en place d'un programme de sélection efficace utilisant ces tests passe par la connaissance de l'hérédité du caractère mesuré. L'estimation des rôles relatifs de l'additivité et de la dominance doit permettre de définir si à partir d'une sélection sur lignées, on peut obtenir des hybrides avec un bon niveau de résistance au $S$. sclerotiorum.

Une telle étude a d'abord été entreprise pour les tests mesurant la résistance à l'extension du mycélium dans les tissus et a porté sur 47 génotypes d'un plan factoriel de croisement. Deux tests de sélection ont permis d'apprécier la résistance de différents tissus. L'héritabilité de ce caractère a été estimée ainsi que les effets relatifs d'additivité et de dominance. Les corrélations entre les données obtenues en laboratoire et les observations d'attaques naturelles en 1985 ont été calculées.

\section{MATÉRIEL ET MÉTHODES}

\section{A. Matériel végétal}

Douze lignées ont été choisies pour être représentatives de la gamme de sensibilité vis-à-vis de $S$. sclerotiorum et de la diversité génétique du tournesol cultivé : B11A3.

- 6 lignées femelles : 62, SD, GH, GU, F10,

La stérilité mâle des 4 premières est cytoplasmique, celle des 2 dernières est génique. Les formes mâlesfertiles ont été utilisées pour les tests sur lignées.

- 6 lignées mâles : Ha61, Rha274, Rha266, V135, Pac1, PRS5.

L'hybride SD $\times$ Rha266 n'a pu être fabriqué. l'étude a donc porté sur 35 hybrides. Parmi ceux-ci, il $\mathrm{y}$ avait des hybrides connus pour leur comportement vis-à-vis de $S$. sclerotiorum et donc pouvant servir de témoins au bon déroulement des tests :

- REMIL (B11A3 × Ha61) - témoin résistant.
- AIRELLE (F10 × V135) ; CR2 (GH × Rha266) - témoins sensibles.

\section{Test sur tige}

Ce test a été proposé par SERIEYS (in VEAR \& TOURVIEILlE, 1984b). Les tiges des plantes cultivées en pot sont sectionnées sous la première paire de vraies feuilles pour les hybrides, sous la deuxième paire pour les lignées. Ainsi, les tiges ont des longueurs équivalentes quel que soit le génotype. Un implant mycélien est inséré dans une fente pratiquée au sommet de la tige. On mesure la vitesse d'extension du champignon dans les tissus de l'hôte à $20^{\circ} \mathrm{C} \pm 2$. Les résultats sont exprimés en $\mathrm{mm} / \mathrm{j}$. Il y a 10 répétitions par génotype.

\section{Test sur capitule}

Ce test a été décrit par VEAR \& GUILlaumin (1976). Trois pastilles de mycélium sont fixées sur la face stérile de chaque capitule maintenu en survie en chambre climatisée (température $18^{\circ} \mathrm{C} \pm 1$ ). Trois jours après l'infection, les taches de pourriture apparues sont mesurées. Les données sont exprimées en surface infectée $\left(\mathrm{mm}^{2}\right)$, moyenne des 3 infections par capitule. Il y a 8 capitules par génotype.

Par suite de la dégénérescence en culture in vitro de la souche employée pour le premier test, une autre souche a été utilisée pour ce deuxième test. En l'absence d'interaction isolats du champignongénotypes de tournesol (VEAR \& GUILLAUMIN, 1977 ; THUAULT, 1986), les réactions des génotypes aux différents tests sont comparables.

\section{B. Observations d'attaques naturelles}

Pour chacune de ces infections, les résultats sont exprimés en pourcentages de plantes malades. Aucune mesure de la sévérité et de l'évolution des symptômes n'est réalisée car ces caractères sont trop dépendants des conditions climatiques pour permettre la comparaison de génotypes présentant des symptômes à des dates différentes.

\section{Infection semi-naturelle}

La méthode décrite par TOURVIEILLE \& VEAR (1984b) a été utilisée pour l'observation des attaques sur capitule. Le dispositif est un essai bloc de FISHER à 4 répétitions et 2 lignes de 23 plantes par répétition. Les résultats sont exprimés en pourcentages de plantes montrant des symptômes.

\section{Attaque naturelle sur bourgeon apical}

Des attaques sur bourgeons se manifestant par une coloration mauve de la pointe des feuilles ont été observées le 15 juin. Le pourcentage de plantes atteintes a été calculé.

\section{Attaque naturelle sur cotylédons}

Pour la première fois, des attaques sur cotylédons ont été observées. Elles se manifestent par des taches rondes de pourriture brune. Si les cotylédons atteints ne sont pas enlevés, la pourriture gagne la tige, puis le collet et provoque la mort des plantes. 


\section{L'hérédité de la résistance : méthodes de calcul}

Toutes les données utilisées sont les moyennes calculées sur les répétitions.

\section{Régression « parent moyen hybride»}

Une régression linéaire est établie entre la valeur de la moyenne des lignées parentales $(\mathrm{X})$ et la valeur de l'hybride correspondant (Y).

La valeur de l'hybride égale à la moyenne de celle de ses parents définit l'additivité stricte. Le coefficient de régression entre ces valeurs permet d'apprécier la part d'additivité intervenant dans le contrôle du caractère. D'autre part, ce coefficient est une estimation de l'héritabilité $\mathrm{h}^{2}$ définie par ailleurs comme le rapport VA/VP (VA : variance d'additivité ; VP variance phénotypique) (FALCONER, 1972).

\section{Régression «parent-descendance »}

Une régression linéaire est calculée entre les valeurs propres des lignées $(\mathrm{X})$ et la moyenne des valeurs des hybrides correspondants (Y). Cette relation estime l'intérêt de sélectionner sur la valeur propre des lignées pour le caractère considéré. C'est une autre estimation de l'additivité.

\section{Estimation des effets relatifs d'additivité et de dominance}

Le rapport des variances d'A.G.C. (aptitude générale à la combinaison) et d'A.S.C. (aptitude spécifique à la combinaison) doit permettre d'apprécier l'importance de l'additivité par rapport à la dominance dans le contrôle du caractère. Il a été calculé comme suit :

$$
\begin{aligned}
& \frac{\text { Var AGC }}{\operatorname{Var} A S C}=\frac{\sigma^{2} \text { AGC }}{\sigma^{2} \text { ASC }} \text { avec } \\
& \sigma^{2} \mathrm{AGC}=\sigma^{2} \mathrm{AGC} q+\sigma^{2} \mathrm{AGC} \sigma^{\circ} \\
& \sigma^{2} \mathrm{AGC}=\frac{\mathrm{CM} Q-\left(\mathrm{CM} Q \times \sigma^{\prime}\right)}{\mathrm{IL}}+ \\
& +\frac{\mathrm{CMO}^{\pi}-\left(\mathrm{CM} Q \times \sigma^{*}\right)}{\mathrm{JL}} \\
& \sigma^{2} \mathrm{ASC}=\frac{\mathrm{CMO} \times \sigma^{*}-\mathrm{CME}}{\mathrm{L}}
\end{aligned}
$$

avec I nombre de mâles

J nombre de femelles

$\mathrm{L}$ nombre de répétitions

et

$$
\begin{aligned}
\mathrm{CM}(q)= & \mathrm{LI} \sum_{j=1}^{\mathrm{J}}(\overline{\mathrm{x}} \mathrm{j}-\overline{\mathrm{x}})^{2} /(\mathrm{J}-1) \\
\mathrm{CM}\left(\sigma^{\prime}\right)= & \mathrm{LJ} \sum_{\mathrm{i}=1}^{1}(\overline{\mathrm{x}} \mathrm{i}-\overline{\mathrm{x}})^{2} /(\mathrm{I}-1) \\
\mathrm{CM}\left(q \times O^{\prime}\right)= & \mathrm{L} \sum_{\mathrm{i}, \mathrm{j}}^{\mathrm{J} J} \times \\
& \times(\overline{\mathrm{x}} \mathrm{ij}-\overline{\mathrm{x}} \mathrm{i}-\overline{\mathrm{x}} \mathrm{j}+\overline{\mathrm{x}})^{2} /(\mathrm{I}-1)(\mathrm{J}-1) \\
\mathrm{CM}_{(\mathrm{E})}= & \sum_{k=1}^{\mathrm{L}}(\mathrm{xijk}-\overline{\mathrm{x}} \mathrm{ij})^{2} / \mathrm{IJ}(\mathrm{L}-1)
\end{aligned}
$$

\section{RÉSULTATS}

Les résultats sont regroupés dans les tableaux 1, 2 et 3 .

\section{A. Réaction aux différents tests}

Le test sur tige présente une assez bonne précision (coefficient de variation $(C . V .=14,2$ p. 100) mais semble seulement moyennement discriminant car l'amplitude entre les données est de $10,7 \mathrm{~mm} / \mathrm{j}$ pour une croissance moyenne de 20,9. Il est corrélé aux attaques sur cotylédons (tabl. 4) mais celles-ci n'ayant été importantes que sur certains génotypes, il faut être prudent dans l'exploitation de ces données.

Le test sur capitule est discriminant mais peu précis $(\mathrm{C} . \mathrm{V} .=42,4$ p. 100$)$, ce manque de précision s'explique peut être par un contact plus ou moins étroit entre le mycélium et l'épiderme du capitule lors de l'inoculation. Cependant, il est corrélé significativement avec les attaques naturelles sur cotylédons, sur bourgeons et sur capitules (tabl. 4).

Les taux d'infection naturelle sur capitule ont été hétérogènes ( $F$ répétition hautement significatif) et extrêmement faibles (1,8 p. 100 en moyenne). Seulement quelques génotypes, principalement des hybrides fabriqués avec V135, GH et GU, présentent des taux d'infection plus élevés, supérieurs à 4 p. 100 (tabl. 2 et 3 ).

Les taux d'attaques sur cotylédons et bourgeons ont été plus élevés sur les hybrides que sur les lignées (tabl. 2). En l'absence de dispositif expérimental avec

\begin{tabular}{|c|c|c|c|c|}
\hline & \multicolumn{2}{|c|}{ Test sur tige $(\mathrm{mm} / \mathrm{j})$} & \multicolumn{2}{|c|}{ Test sur capitule $\left(\mathrm{mm}^{2}\right)$} \\
\hline & $\begin{array}{l}\text { Valeur } \\
\text { propre }\end{array}$ & $\begin{array}{c}\text { Moyenne } \\
\text { des } \\
\text { hybrides }\end{array}$ & $\begin{array}{l}\text { Valeur } \\
\text { propre }\end{array}$ & $\begin{array}{c}\text { Moyenne } \\
\text { des } \\
\text { hybrides }\end{array}$ \\
\hline \multicolumn{5}{|c|}{ Lignées femelles } \\
\hline $\mathrm{B} 11 \mathrm{~A} 3$ & 16,8 & 19,4 & 843,2 & 1213,1 \\
\hline 62 & 23,2 & 23,6 & 440,4 & 950,9 \\
\hline SD & 15,9 & 21,1 & 417,4 & 826,4 \\
\hline $\mathrm{GH}$ & 17,5 & 22,9 & 1554,4 & 1491,2 \\
\hline GU & 24,4 & 23,9 & 1157,7 & 1300,6 \\
\hline F10 & 19,9 & 21,4 & 342,0 & 874,4 \\
\hline \multicolumn{5}{|c|}{ Lignées mâles } \\
\hline Ha61 & 16,6 & 19,4 & 343,7 & 942,0 \\
\hline Rha274 & 16,6 & 21,8 & 802,9 & 1180,8 \\
\hline Rha266 & 18,1 & 23,0 & 957,4 & 1327,5 \\
\hline V135 & 19,1 & 23,2 & 1553,6 & 1596,2 \\
\hline Pacl & 20,6 & 23,1 & 279,1 & 1051,5 \\
\hline PRS5 & 23,0 & 22,8 & 208,7 & 641,9 \\
\hline
\end{tabular}
répétitions, seuls les effets parentaux ont pu être étudiés. Il existe un effet mâle significatif pour les 2 types d'attaque. L'effet femelle, plus faible, n'est significatif que pour les attaques sur bourgeon (tabl. 5).

TABLEAU 1

Valeurs propres des lignées et moyennes des hybrides pour 2 tests mycéliens. Results of two mycelial tests on inbred lines and the means of their corresponding hybrids. 
TABLEAU 2

Valeurs propres des lignées et moyennes des hybrides pour 3 attaques naturelles.

Observations of 3 types of natural attack on inbred lines and their corresponding hybrids.

\begin{tabular}{|c|c|c|c|c|c|c|}
\hline & \multicolumn{2}{|c|}{ Attaques sur cotylédons $(\%)$} & \multicolumn{2}{|c|}{ Attaques sur bourgeon $(\%)$} & \multicolumn{2}{|c|}{ Attaques sur capitule $(\%)$} \\
\hline & $\begin{array}{l}\text { Valeur } \\
\text { propre }\end{array}$ & $\begin{array}{l}\text { Moyenne des } \\
\text { hybrides }\end{array}$ & $\begin{array}{l}\text { Valeur } \\
\text { propre }\end{array}$ & $\begin{array}{l}\text { Moyenne des } \\
\text { hybrides }\end{array}$ & $\begin{array}{l}\text { Valeur } \\
\text { propre }\end{array}$ & $\begin{array}{c}\text { Moyenne des } \\
\text { hybrides }\end{array}$ \\
\hline \multicolumn{7}{|c|}{ Lignées femelles } \\
\hline $\mathrm{B} 11 \mathrm{~A} 3$ & 0,7 & 1,7 & 5,5 & 49,4 & 0,0 & 0,1 \\
\hline 62 & 8,0 & 1,9 & 9,4 & 41,1 & 1,5 & 0,5 \\
\hline SD & 0,0 & 2,6 & 0,0 & 9,7 & 0,0 & 0,6 \\
\hline $\mathrm{GH}$ & 0,0 & 5,3 & 0,0 & 47,4 & 3,2 & 2,1 \\
\hline GU & 8,3 & 3,4 & 0,0 & 22,4 & 0,0 & 1,9 \\
\hline F10 & 0,0 & 3,6 & 0,0 & 2,6 & 0,0 & 2,0 \\
\hline \multicolumn{7}{|c|}{ Lignées mâles } \\
\hline Ha61 & 0,0 & 0,1 & 0,0 & 32,4 & 0,7 & 0,5 \\
\hline Rha274 & 0,8 & 0,7 & 11,0 & 66,1 & 0,0 & 1,3 \\
\hline Rha266 & 0,0 & 0,0 & 0,0 & 30,7 & 0,0 & 1,0 \\
\hline V135 & 4,2 & 8,8 & 4,4 & 24,7 & 0,0 & 3,4 \\
\hline Pacl & 0,0 & 3,8 & 0,0 & 14,2 & 0,0 & 0,6 \\
\hline PRS5 & 0,0 & 3,6 & 0,0 & 3,6 & 0,0 & 1,0 \\
\hline
\end{tabular}

TABLEAU 3

Résultats de 2 tests mycéliens sur 35 hybrides. Results of 2 mycelial tests on 35 hybrids.

\begin{tabular}{llcr}
\hline \hline & $\begin{array}{c}\text { Test sur } \\
\text { tige } \mathrm{mm} / \mathrm{j}\end{array}$ & $\begin{array}{c}\text { Test sur } \\
\text { capitule } \mathrm{mm}^{2}\end{array}$ \\
\hline Hybrides & & \\
B11A3 $\times$ Ha61 & & \\
62 & $\times$ Ha61 & 17,8 & 1097,2 \\
SD & $\times$ Ha61 & 19,0 & 640,8 \\
GH & $\times$ Ha61 & 17,6 & 844,5 \\
GU & $\times$ Ha61 & 23,4 & 956,4 \\
F10 & $\times$ Ha61 & 22,4 & 1030,3 \\
B11A3 $\times$ Rha274 & 15,9 & 1082,8 \\
62 & $\times$ Rha274 & & 1165,7 \\
SD & $\times$ Rha274 & 23,6 & 1583,4 \\
GH & $\times$ Rha274 & 22,5 & 733,4 \\
GU & $\times$ Rha274 & 22,9 & 1265,4 \\
F10 & $\times$ Rha274 & 22,7 & 1529,2 \\
B11A3 $\times$ Rha266 & 17,1 & 808,0 \\
62 & $\times$ Rha266 & 19,8 & 1331,5 \\
GH $\times$ Rha266 & 25,7 & 1461,0 \\
GU & $\times$ Rha266 & 22,4 & 1361,2 \\
F10 & $\times$ Rha266 & 24,2 & 1579,1 \\
B11A3 $\times$ V135 & & 904,8 \\
62 & $\times$ V135 & 20,5 & 1568,2 \\
SD $\times$ V135 & 26,6 & 1335,3 \\
GH $\times$ V135 & 20,9 & 1826,3 \\
GU $\times$ V135 & 21,2 & 2104,9 \\
F10 & $\times$ V135 & 24,6 & 1133,6 \\
B11A3 $\times$ Pac1 & 25,6 & 1609,1 \\
62 & $\times$ Pac1 & 17,2 & 1290,8 \\
SD $\times$ Pac1 & 24,5 & 535,4 \\
GH $\times$ Pac1 & 23,3 & 459,2 \\
GU $\times$ Pac1 & 23,6 & 2138,7 \\
F10 $\times$ Pac1 & 24,9 & 1496,5 \\
B11A3 $\times$ PRS5 & 24,9 & 388,7 \\
62 & $\times$ PRS5 & 21,5 & 825,5 \\
SD $\times$ PRS5 & 22,2 & 149,3 \\
GH $\times$ PRS5 & 21,1 & 268,4 \\
GU $\times$ PRS5 & 24,0 & 1120,6 \\
F10 $\times$ PRS5 & 24,5 & 1034,7 \\
\hline \hline & & 23,3 & 453,3 \\
\hline
\end{tabular}

TABLEAU 4

Corrélations entre les résultats obtenus sur les hybrides au cours des tests et en attaques naturelles.

Correlations between tests and natural attacks.

\begin{tabular}{lcccr}
\hline \hline & $\begin{array}{c}\text { Test } \\
\text { sur } \\
\text { tige }\end{array}$ & $\begin{array}{c}\text { Attaque } \\
\text { sur } \\
\text { capitule }\end{array}$ & $\begin{array}{c}\text { Attaque } \\
\text { sur } \\
\text { bourgeon }\end{array}$ & $\begin{array}{c}\text { Attaque } \\
\text { sur } \\
\text { cotylédon }\end{array}$ \\
\hline Test sur capitule & 0,164 & $0,355^{*}$ & $0,336^{*}$ & $0,435^{*}$ \\
Test sur tige & & 0,277 & 0,054 & $0,481^{*}$ \\
Attaque sur capitule & & & 0,079 & $0,453^{*}$ \\
Attaque sur bourgeon & & & & $-0,035$ \\
\hline \hline
\end{tabular}

* Significatif au seuil $5 \%$.

\section{TABLEAU 5}

Effets femelle et mâle calculés à partir de l'analyse de variance pour les attaques sur cotylédons et bourgeons (DDL $\left.\left(N_{1} / N_{2}\right): 5 / 24\right)$.

Female and male effects from the variance analysis for natural attack on cotyledons and buds (DF $\left.\left(N_{I} / N_{2}\right): 5 / 24\right)$.

\begin{tabular}{lcc}
\hline \multirow{2}{*}{ Effets } & Sur cotylédons & Sur bourgeons \\
\cline { 2 - 3 } & $\mathrm{Fc}$ & $\mathrm{Fc}$ \\
\hline Femelle & $2,57 \mathrm{NS}$ & $4,89^{* *}$ \\
Mâle & $5,53^{* *}$ & $7,97^{* * *}$ \\
\hline \hline
\end{tabular}

NS : Non significatif à $5 \%$.

** : Significatif à $1 \%$.

*** : Significatif à $0,1 \%$.

\section{B. Etude de l'hérédité de la résistance}

Les analyses de variances sur les données brutes des hybrides aux 2 tests montrent dans tous les cas un F génotype hautement significatif (tabl. 6). Il paraît 
TABLEAU 6

Effet génotype calculé à partir de l'analyse de variance pour 2 tests mycéliens et attaque sur capitule.

Genotype effect from the variance analysis for 2 mycelial tests and natural attack on head.

\begin{tabular}{lccc}
\hline \hline & $\begin{array}{c}\text { Test } \\
\text { sur tige }\end{array}$ & $\begin{array}{c}\text { Test } \\
\text { sur capitule }\end{array}$ & $\begin{array}{c}\text { Attaque } \\
\text { sur capitule }\end{array}$ \\
\hline ddl $\left(\mathrm{n}_{1} / \mathrm{n}_{2}\right)$ & $(31 / 266)$ & $(34 / 245)$ & $(35 / 105)$ \\
F hybride & 5,18 & 9,14 & 2,37 \\
& $* * *$ & $* * *$ & $*$ \\
\hline
\end{tabular}

* : Significatif à $5 \%$.

*** : Significatif à $0,1 \%$.

donc possible de trouver des génotypes plus résistants et d'obtenir un progrès génétique pour la résistance à S. sclerotiorum.

Les régressions $R$ (parent moyen-hybride) et $R^{\prime}$ (parent-descendance) sont hautement significatives pour le test sur capitule ( $F$ régression $=43,6$ et 59,7 respectivement), significatives pour le test sur tige (F régression $=5,4$ et 8,9 ).

Le test sur capitule montre des régressions plus élevées $\left(R=1,105 ; R^{\prime}=0,555\right)$ que le test sur tige $\left(\mathrm{R}=0,843 ; \mathrm{R}^{\prime}=0,362\right.$ ) (tabl. 7). Il ne nous est pas possible de comparer les rapports Var AGC/Var ASC à cause de la perte d'un nombre assez important de plantes pour le test sur tige ; le modèle expérimental, devenu non orthogonal, n'a pas permis une analyse statistique simple des données. Le rapport Var AGC/ Var ASC est faible $(1,24)$ pour le test sur capitule.

Les résultats dont nous disposons (coefficients des différentes régressions linéaires) laissent supposer une additivité plus importante au niveau du capitule qu'au niveau de la jeune tige et il paraît plus facile de prédire le comportement des hybrides à partir de celui des lignées parentales, avec le test sur capitule.

\section{DISCUSSION ET CONCLUSION}

\section{A. Qualité des tests}

\section{Test sur tige}

SERIEYS (in VEAR \& TOURVIEILLE, 1984b) a trouvé que le test sur tige était un bon prédicteur de la sensi- bilité aux attaques sur bourgeon apical. Cette constatation est opposée à celle que nous avons faite. Néanmoins, les attaques sur bourgeon relevées à ClermontFerrand n'ayant pas évolué en pourriture molle, les symptômes observés ne sont pas les mêmes que ceux décrits par SERIEYS ; il n'est donc pas certain qu'il y ait réellement contradiction. Il est nécessaire de maîtriser ce type de contamination afin d'obtenir des résultats reproductibles et fiables. Ce test pourrait être utilisable en sélection mais il reste à définir sa signification en ce qui concerne la résistance aux différentes formes d'attaque de S. sclerotiorum.

\section{Test sur capitule}

VEAR \& GUILlAUMIN (1977) ont mis en évidence, sur 26 génotypes, une corrélation positive entre les résultats du test sur capitule et le comportement en plein champ de ces génotypes; par contre, TouRVIEILLE \& VEAR (1984a) ne retrouvent pas ce résultat en travaillant sur 9 hybrides différents. En 1985, l'existence d'une corrélation avec les attaques sur capitules, observées en conditions semi-contrôlées, donc fiables, (TOURVIEILLE \& VEAR, 1984b), laisse supposer que ce test juge correctement les génotypes présentant une résistance ou une sensibilité bien marquée.

Les corrélations entre les infections par implant mycélien sur capitule et les attaques naturelles sur organes juvéniles (cotylédons et bourgeons apicaux) s'expliquent peut-être par une pénétration facile du parasite dans les tissus jeunes qui, en réduisant la phase saprophytique, se rapprocherait d'une infection par implant mycélien.

La corrélation la plus forte observée avec les attaques sur cotylédons est peut-être liée aux dégâts dus au gel (température inférieure à $-4^{\circ} \mathrm{C}$ en mai). Les parties nécrosées auraient servi de support nutritif ayant favorisé le développement du mycélium et donc de l'infection, au même titre que la gélose maltée du test.

\section{B. Hérédité de la résistance}

Il existe une variation continue des valeurs obtenues pour chacun des tests sur l'ensemble des 47 génotypes. Un même génotype peut apparaître comme sensible ou résistant selon les tests employés et selon les orga-

TABLEAU 7

Coefficients de régression entre lignées et hybrides, rapport Var AGC/Var ASC.

Regression coefficients between inbred lines and hybrids and Var GCA/Var SCA.

\begin{tabular}{|c|c|c|c|}
\hline \multirow{2}{*}{ Tests } & \multirow{2}{*}{ Régression parent moyen-hybride } & \multirow{2}{*}{ Régression parent-descendance } & Var AGC \\
\hline & & & Var ASC \\
\hline Capitule & $1,105 \pm 0,335^{* * *}$ & $0,555 \pm 0,156^{* * *}$ & 1,24 \\
\hline Tige & $0,843 \pm 0,443^{* * *}$ & $0,362 \pm 0,287^{*}$ & - \\
\hline
\end{tabular}

* : Significatif au seuil $5 \%$.

*** : Significatif au seuil $0,1 \%$. 
nes attaqués. Les valeurs calculées pour apprécier l'héritabilité de la résistance au $S$. sclerotiorum varient selon le test appliqué. Cette étude confirme le caractère polygénique et complexe de la résistance mise en évidence chez différentes espèces (FULLER et al., 1984 ; PIRVU et al., 1985).

\section{Test sur tige}

Les régressions parent moyen-hybride et parentdescendance sont significatives ; l'additivité intervient de façon importante car le coefficient d'héritabilité est élevé $(0,843 \pm 0,443)$ mais d'après l'étude des données brutes (ROBERT, 1985), il est probable qu'intervient une part d'interactions de dominance à ne pas négliger.

Les variations relatives autour des valeurs moyennes des coefficients de régression parent moyen-hybride (R) et parent-descendance $\left(R^{\prime}\right)$ sont élevées $\left(52,5\right.$ p. 100 pour $R$ et 79,5 p. 100 pour $\left.R^{\prime}\right)$; les coefficients de corrélation, bien que significatifs, ne sont pas très élevés $(r=0,57$ et $r=0,69$ respectivement) (fig. 1 et 2). Deux hypothèses peuvent expliquer cette

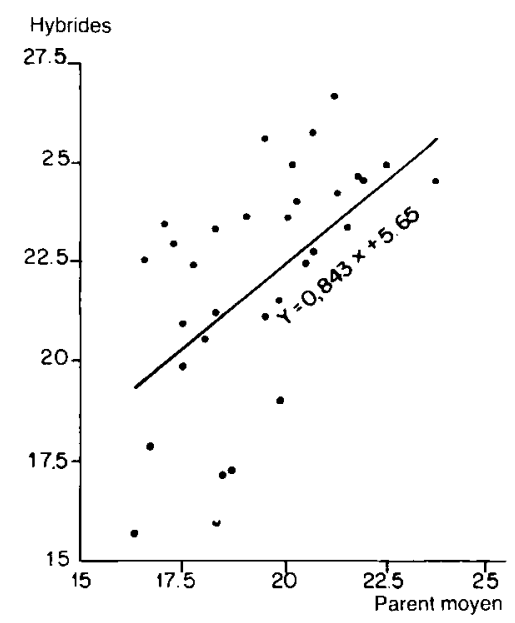

Figure 1

Test sur tige : régression parent moyen/hybrides (en $\mathrm{mm} / \mathrm{j}$ ). Stem test : mid-parent/hybrids regression (units are $\mathrm{mm} /$ day).

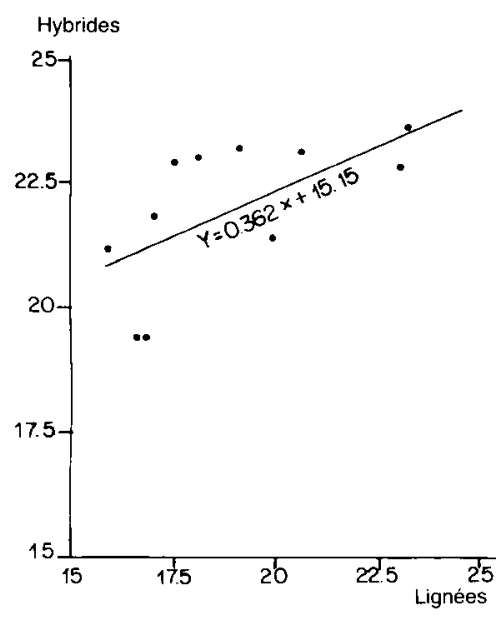

Figure 2

Test sur tige : régression parent/descendance (en $\mathrm{mm} / \mathrm{j}$ ).

Stem test : parent/progeny regression (units are $\mathrm{mm} /$ day). dispersion. D'une part, les génotypes parentaux ont été choisis pour être représentatifs de la variabilité du tournesol cultivé pour la réponse à l'infection par $S$. sclerotiorum sur capitule; ils représentent peut-être une gamme différente de résistance au niveau de la tige. D'autre part, les réponses des jeunes plantes plus ou moins vigoureuses, ne sont pas homogènes et les résultats des tests ne seraient peut-être pas des mesures exactes de la résistance génotypique.

Il serait intéressant de répéter l'expérience sur deux groupes distincts de lignées : l'un composé de lignées résistantes, l'autre de lignées sensibles; les niveaux moyens des hybrides obtenus, les coefficients d'héritabilité et leur dispersion permettraient de préciser s'il est possible de sélectionner des hybrides exprimant une résistance, en utilisant ce test.

\section{Test sur capitule}

Les deux régressions calculées sont très hautement significatives (tabl. 7 , fig. 3 et 4). L'additivité doit jouer un rôle important dans la résistance aux attaques sur capitule, car le coefficient de régression

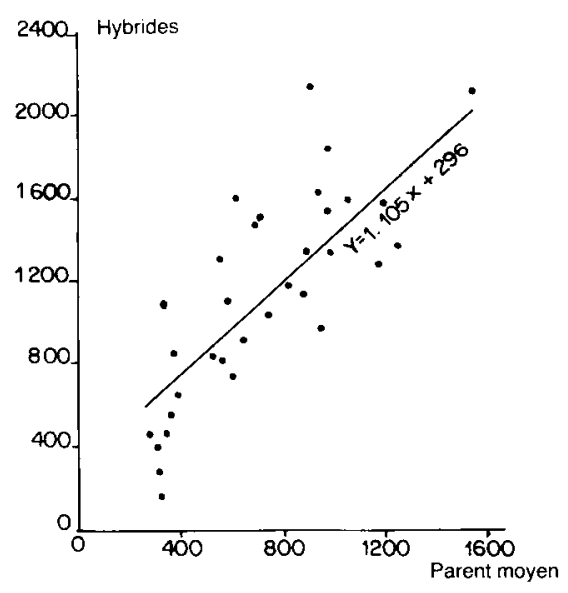

Figure 3

Test sur capitule : régression parent moyen/hybrides (en $\mathrm{mm}^{2}$ ). Capitulum test : mid-parent/hybrid regression (units are $\mathrm{mm}^{2}$ ).

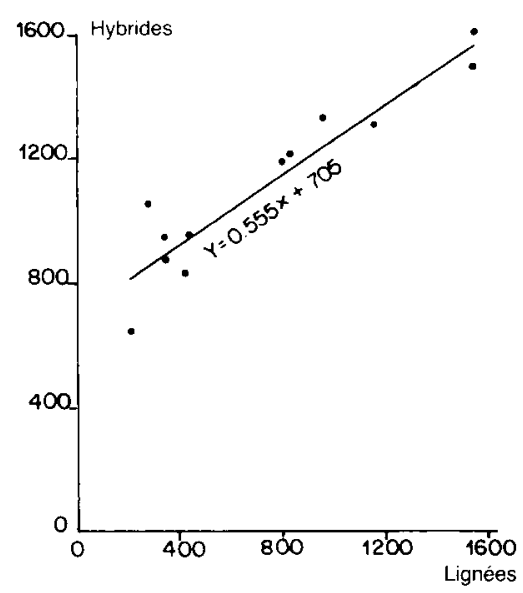

Figure 4

Test sur capitule : régression parent/descendance (en $\mathrm{mm}^{2}$ ).

Capitulum test : parent/progeny regression (units are $\mathrm{mm}^{2}$ ). 
parent moyen-hybride est élevé $(1,105 \pm 0,335)$ et sa variation relative est moyenne (30 p. 100) (fig. 3). La surface moyenne des taches, plus grande chez les hybrides que chez les lignées ainsi qu'une amplitude 1,5 fois plus importante, expliquent que l'on obtienne un coefficient de régression supérieur à 1 (tabl. 1 et 3). Vu l'intervalle de confiance de ce coefficient, on peut considérer que l'héritabilité dépasse 0,77 . Ces résultats sont en accord avec ceux obtenus sur tournesol par VEAR \& GUILLAUMIN (1977) et sur haricot par FULLER et al. (1984).

La corrélation parent-descendance étant élevée $(0,93)$ et la régression parent-descendance forte, la connaissance de la valeur propre d'une lignée semble permettre une bonne prédiction de sa valeur en combinaison et implique de sélectionner sur la valeur propre des lignées. Cependant, le rapport Var AGC/Var ASC étant de 1,24, il ne faut pas négliger les effets de dominance.

FICK (1975) a calculé sur des descendances $F_{2}, F_{3}$ et des rétrocroisements une héritabilité pour la teneur en huile égale à 0,61 . A partir de 12 parents (différents de ceux cultivés par FICK), 35 hybrides et 8 répétitions, nous avons trouvé une héritabilité pour la résistance à $S$. sclerotiorum plus importante (supérieure à 0,77 ). Comme pour la teneur en huile, il est envisageable de sélectionner pour la résistance à Sclerotinia au niveau des générations précoces sur individus et également sur famille (VEAR résultats non publiés).

Cependant, ce test ne peut pas faire ressortir tous les facteurs de résistance des génotypes au $S$. sclerotio- rum du capitule. Pour étudier le comportement aux premières phases de l'infection par ascospores, il faut utiliser un autre test plus proche des phénomènes de contaminations naturelles: le test "ascospores» (VEAR \& TOURVIEILlE, 1984a). La mise en œuvre simultanée de ces deux tests permettrait de juger plusieurs facteurs de résistance. Il est donc envisagé, sur ce même matériel, de réaliser des tests par ascospores ainsi que des tests jugeant du comportement du tournesol aux attaques racinaires afin de mieux appréhender l'hérédité de la résistance au cours de ces différentes phases de l'infection et la meilleure utilisation des tests.

Toute stratégie de sélection pour la résistance au $S$ sclerotiorum doit tenir compte de cette complexité de la résistance et du besoin, à long terme, de rassembler des gènes de résistance à chaque forme d'attaque. Ainsi il semble nécessaire de réaliser une sélection récurrente sur populations qui favorise les recombinaisons. Employés pendant cette sélection et pendant la phase suivante de création des lignées, les tests de résistance devraient permettre de faire ressortir les ensembles de gènes les plus intéressants.

Reçu le 8 juillet 1986. Accepté le 14 mars 1987.

\section{REMERCIEMENTS}

Les auteurs tiennent à remercier Messieurs BRIAN et VINCOURT pour leur aide apportée aux traitements statistiques des données.

\section{RÉFÉRENCES BIBLIOGRAPHIQUES}

Falconer D. S., 1972. Introduction à la génétique quantitative. Masson et Cie, $284 \mathrm{p}$.

Fick G. N., 1975. Heritability of oil content in sunflowers. Crop Science, 15, 77-78.

Fuller P., Coyne D. P., Steadman J. R., 1984. Inheritance of resistance to white mold disease in a diallel cross of dry beans. Crop Science, 24, 929-933.

Lamarque C., Tourvieille D., Regnault T. Y., Pierre J. C., 1985. Les problèmes posés par la lutte chimique contre le Sclerotinia sclerotiorum sur capitule de tournesol. In Premières Journées d'études sur les maladies des plantes. A.N.P.P., 2, 359-367.

Pirvu N., Vranceanu A. V., Stoenescu F., 1985. Genetic mechanisms of sunflower resistance to white rot (Sclerotinia sclerotiorum (Lib.) de Bary). Z. Pflanzenzüchtg, 95, 157-163.

Purdy L. H., 1979. Sclerotinia sclerotiorum : history, diseases and symptomatology, host range, geographic distribution and impact. Phytopathology, 69, 875-880.

Robert N., 1985. Approche de l'hérédité des caractères de résistance à Sclerotinia sclerotiorum chez le tournesol. Etude comparative de tests de sélection. D.E.A. d'Agronomie, Université de Rennes I, France, $68 \mathrm{p}$.
Thuault M. C., 1986. Etudes des relations hôte-parasite chez le couple Helianthus annuus - Sclerotinia. D.E.A. de Biologie fondamentale et appliquée, Université de Clermont-Ferrand, France, 74 p.

Tourvieille D., Vear F., 1984a. Comparaison de méthodes d'estimation de la résistance du tournesol à Sclerotinia sclerotiorum. (Lib.) de Bary, Agronomie, 4 (6), 517-525.

Tourvieille D., Vear F., 1984b. La sélection du tournesol pour une meilleure résistance au Sclerotinia sclerotiorum. Inf. techn. CETIOM, 88, 3-23.

Vear F., Guillaumin J. J., 1976. Development of a test useable in breeding for resistance to sunflower capitulum attack by Sclerotinia sclerotiorum, Proc. 7 th Intern. Sunflower Conference, Krasnodar, U.R.S.S., 1, 205-212.

Vear F., Guillaumin J. J., 1977. Etude de méthodes d'inoculation du tournesol par Sclerotinia sclerotiorum et application à la sélection. Ann. Amélior. Plantes, 27 (5), 523-537.

Vear F., Tourvieille D., 1984a. Recurrent selection for resistance to Sclerotinia sclerotiorum in sunflowers using artificial infections. Agronomie, 4 (8), 789-794.

Vear F., Tourvieille D., 1984b. Sclerotinia sclerotiorum sur tournesol. Différences de sensibilité variétale et méthodes de sélection. Conférence du 28 février 1984, I.N.R.A., Clermont-Ferrand, 106 p. 